\title{
Vibrational energies of some diatomic molecules for a modified and deformed potential
}

\section{A. Onate ( $\square$ oaclems14@physicist.net)}

Landmark University

\section{B. Okon}

University of Uyo

\author{
M. C. Onyeaju \\ University of Port Harcourt \\ O. Ebomwonyi \\ University of Benin
}

\section{Research Article}

Keywords: Eigensolution, Bound state, Wave equation, Schrödinger equation, Diatomic molecules

Posted Date: September 15th, 2021

DOI: https://doi.org/10.21203/rs.3.rs-892967/v1

License: (c) (1) This work is licensed under a Creative Commons Attribution 4.0 International License.

Read Full License

Version of Record: A version of this preprint was published at Scientific Reports on November 18th, 2021. See the published version at https://doi.org/10.1038/s41598-021-01998-6. 
Vibrational energies of some diatomic molecules for a modified and deformed potential

\author{
C.A.Onate, ${ }^{*}$, I.B. Okon ${ }^{2}$, M.C. Onyeaju ${ }^{3}$ and O. Ebomwonyi ${ }^{4}$. \\ ${ }^{1}$ Physics Programme, Department of Physical Sciences, Landmark University, Omu-Aran, Nigeria \\ ${ }^{2}$ Theoretical Physics Group, Department of Physics, University of Uyo, Nigeria \\ ${ }^{3}$ Theoretical Physics group, Department of Physics, University of Port Harcourt, P.M.B. 5323 Choba, Port \\ Harcourt-Nigeria. \\ ${ }^{4}$ Department of Physics, University of Benin, Benin City, Nigeria.
}

\begin{abstract}
A molecular potential model is proposed and the solutions of the radial Schrödinger equation in the presence of the proposed potential is obtained. The energy equation and its corresponding radial wave function are calculated using the powerful parametric Nikiforov-Uvarov method. The energies of cesium dimer for different quantum states were numerically obtained for both negative and positive values of the deformed and adjustable parameters. The results for sodium dimer and lithium dimer were calculated numerically using their respective spectroscopic parameters. The calculated values for the three molecules are in excellent agreement with the observed values. Finally, we calculated different expectation values and examined the effects of the deformed and adjustable parameters on the expectation values.
\end{abstract}

Corresponding Author: "oaclems14@physicist.net.

Key words: Eigensolution; Bound state; Wave equation; Schrödinger equation; Diatomic molecules.

\title{
1. Introduction
}

In the recent time, exponential-type potential has been the subject of interest in the quantum mechanics which greatly popularized the relativistic and non-relativistic wave equations such as the Schrödinger equation, Klein-Gordon equation, Dirac equation and others [1-15]. The approximate solutions of these wave equations have been obtained mostly for one-dimensional system with various exponential-type potentials using different approximation methods developed by different authors. The frequently used methods are Nikiforov-Uvarov method [16, 17], asymptotic iteration method [18], supersymmetric approach [19, 20], factorization method 21], exact and proper quantization rule [22, 23]. Recently, Ikot et al. [24, 25] have used a new approach called NU Functional analysis method. The different methods have different approach for the solutions of the wave equations but give results that are approximately the same. For instance, the solutions of the radial Schrödinger equation under the Deng-Fan potential model has been studied by Dong and Gu [26] using factorization method. Zhang et al. [27] and Onate et al. [28] respectively also studied the potential via supersymmetry quantum mechanics and parametric Nikiforov-Uvarov method respectively. The respective result of these authors agreed with one another.

The solutions of the wave equations studied for different potentials, have been applied to the study of several systems such as Theoretic quantities [29-32] and Thermal properties (mean energy, heat capacity, free energy and entropy) [33-38]. In ref. [27], the result was used to study the rotation transition frequency for HF. In ref. [28], the wave function was used to study some theoretic quantities such Shannon entropy and Rényi entropy. In ref. [39], the problem of $s o(2,2)$ was studied under the Pöschl-Teller potential. Several authors have also studied the 
energy eigenvalues for many diatomic molecules on molecular dynamics and spectroscopy in the field of chemistry and molecular physics [40, 41]. This provides explanations about the dynamics and physical properties of some molecules. The potential energy function involved are used to study the bonding between atoms, hence the predictions to the behaviour of some class of molecules [42]. Some of these potentials can be used to describe some experimental values. Generally, a good empirical internuclear potential function should reproduce the experimental potential energy curves as determined by the RKR method. Considering this, the present study wants to examine an approximate solutions of the Schrödinger equation with a new modified and deformed exponential-type molecular potential model confined on a cesium dimer, sodium dimer and lithium dimer. The study also aim to investigate the potential with two different values for each of the deformed parameter and adjustable parameter under the same cesium dimer. This potential has not been reported for any study yet to the best our understanding.

The cesium dimer is an important molecule that has many applications, e.g. vibrational cooling of molecules, population dynamics, and even coherent control [43-47]. The cesium molecule is an attractive system for examining a possible variation of the electron-to-proton mass ratio and of the fine-structure constant [48]. It is noted that $3^{3} \sum_{g}^{+}$state of cesium dimer has a strong Fermi contact interaction with the nuclei, and possesses a large hyperfine splitting [49]. The potential energy curve of the cesium dimer for $3^{3} \sum_{g}^{+}$and $a^{3} \sum_{u}^{+}$states has been reported in ref. $[50,51]$. The modified and deformed exponential-type molecular potential model is given as

$$
V(r)=D_{e}-\frac{D_{e}}{C e^{-\alpha r}+q_{0}}\left(e^{\alpha r_{e}}+q_{1}-\frac{\left(e^{\alpha r_{e}}+q_{1}\right)^{2}}{C e^{-\alpha r}+q_{0}}\right),
$$

where $C$ is a modified parameter, $q_{0}$ is a deformed parameter and $q_{1}$ is an adjustable parameters whose value can be taken as \pm 1 . When the value of the adjustable parameter equals the value of the deformed parameter within \pm 1 , the results of potential (1) gives other useful results. $D_{e}$ is the dissociation energy $r_{e}$ is the equilibrium bond separation and $\alpha$ is the screening parameter. Its numerical value can be obtain using the formula

$$
\alpha=\pi c \omega_{e} \sqrt{\frac{2 \mu}{D_{e}}}+\frac{1}{r_{e}} W\left(-\pi c \omega_{e} \sqrt{\frac{2 \mu}{D_{e}}} e^{-\pi c \omega_{e} \sqrt{\frac{2 \mu}{D_{e}}}}\right),
$$

where $W$ is the Lambert function, $\mu$ is the reduced mass, $c$ is the speed of light and $\omega_{e}$ is the vibrational frequency.

\section{Parametric Nikiforov-Uvarov Method.}

The parametric Nikiforov-Uvarov method is one of the shortest and accurate traditional techniques to solve bound state problems. This method was derived from the conventional Nikiforov-Uvarov method by Tezcan and Sever [17]. According to the authors, the reference equation for the parametric Nikiforov-Uvarov is given as 


$$
\left(\frac{d^{2}}{d s^{2}}+\frac{\alpha_{1}-\alpha_{2}}{s\left(1-\alpha_{3} s\right)} \frac{d}{d s}+\frac{-\xi_{1} s^{2}+\xi_{2} s-\xi_{3}}{s^{2}\left(1-\alpha_{3} s\right)^{2}}\right) \psi(s)=0 .
$$

Following the work of these authors, the condition for eigenvalues equation and wave function are respectively given by $[17,52]$

$$
\begin{gathered}
n \alpha_{2}-(2 n+1) \alpha_{5}+\alpha_{7}+2 \alpha_{3} \alpha_{8}+n(n-1) \alpha_{3}+(2 n+1) \sqrt{\alpha_{9}}+\left(2 \sqrt{\alpha_{9}}+\alpha_{3}(2 n+1)\right) \sqrt{\alpha_{8}}=0, \\
\psi_{n, 1}(s)=N_{n, 1} s^{\alpha_{12}}\left(1-\alpha_{3} s\right)^{-\alpha_{12}-\frac{\alpha_{13}}{\alpha_{3}}} \times P_{n}^{\left(\alpha_{10}-1, \frac{\alpha_{11}}{\alpha_{3}}-\alpha_{10}-1\right)}\left(1-2 \alpha_{3} s\right),
\end{gathered}
$$

The parametric constants in equations (3) and (4) are deduced as follows

$$
\left.\begin{array}{l}
\alpha_{4}=\frac{1-\alpha_{1}}{2}, \alpha_{5}=\frac{\alpha_{2}-2 \alpha_{3}}{2}, \alpha_{6}=\alpha_{5}^{2}+\xi_{1}, \alpha_{7}=2 \alpha_{4} \alpha_{5}-\xi_{2}, \alpha_{8}=\alpha_{4}^{2}+\xi_{3}, \\
\alpha_{9}=\alpha_{3}\left(\alpha_{7}+\alpha_{3} \alpha_{8}\right)+\alpha_{6}, \alpha_{10}=\alpha_{1}+2 \alpha_{4}+2 \sqrt{\alpha_{8}}, \alpha_{11}=\alpha_{2}-2 \alpha_{5}+2\left(\sqrt{\alpha_{9}}+\alpha_{3} \sqrt{\alpha_{8}}\right), \\
\alpha_{12}=\alpha_{4}+\sqrt{\alpha_{8}}, \alpha_{13}=\alpha_{5}-\left(\sqrt{\alpha_{9}}+\alpha_{3} \sqrt{\alpha_{8}}\right)
\end{array}\right\} .
$$

\section{The radial Schrödinger equation and the interacting potential.}

To obtain the energy eigenvalues of the Schrödinger equation with potential (1), we consider the original Schrödinger equation given by

$$
\left[-\frac{\mathrm{h}^{2}}{2 m}\left(\frac{1}{r^{2}} \frac{\partial}{\partial r} r^{2} \frac{\partial}{\partial r}+\frac{1}{r^{2} \sin \theta} \frac{\partial}{\partial \theta}\left(\sin \theta \frac{\partial}{\partial \theta}\right)+\frac{1}{r^{2} \sin ^{2} \theta} \frac{\partial^{2}}{\partial \phi^{2}}\right)+V(r)-E\right] \psi(r)=0 .
$$

Setting the wave function $\psi(r)=U_{n, 1}(r) Y_{m, 1}(\theta, \phi) r^{-1}$, and consider the radial part of the Schrödinger equation, Eq. (7) becomes

$$
-\frac{\mathrm{h}^{2}}{2 \mu} \frac{d^{2} U_{n \mathrm{l}}(r)}{d r^{2}}+\frac{\mathrm{h}^{2}}{2 \mu} \frac{1(1+1)}{r^{2}} U_{n \mathrm{l}}(r)=E_{n \mathrm{l}} U_{n \mathrm{l}}(r)-V(r) U_{n \mathrm{l}}(r),
$$

where $\frac{\mathrm{h}^{2}}{2 \mu} \frac{1(1+1)}{r^{2}}$ has been introduced, $V(r)$ is the interacting potential given in Eq. (1), $E_{n 1}$ is the non-relativistic energy of the system, his the reduced Planck's constant, $m$ is the mass, $n$ is the quantum number, $U_{n l}(r)$ is the wave function. To overcome the centrifugal term in Eq. (7), the following approximation scheme is employed

$$
\frac{1}{r^{2}} \approx \alpha^{2}\left(C_{0}+\frac{e^{\alpha r}}{\left(1+q_{0} e^{\alpha r}\right)^{2}}\right)
$$


For the approximation scheme to suit the interacting potential, the modified parameter is taken to be unity. Substituting Eq. (1) and Eq. (9) into (8), and by defining $y=\frac{1}{e^{-\alpha r}}$, the radial Schrödinger equation with the deformed exponential-type potential turns to be

$$
\frac{d^{2} U_{n \mathrm{l}}(y)}{d y^{2}}+\frac{1+y}{y\left(1+q_{0} y\right)} \frac{d U_{n \mathrm{l}}(y)}{d y}+\frac{-P y^{2}+Q y-R}{y^{2}\left(1+q_{0} y\right)^{2}} U_{n \mathrm{l}}(y)=0
$$

where

$$
\begin{aligned}
& P=\frac{2 \mu D_{e}}{\alpha^{2} \mathrm{~h}^{2}}\left(q_{0}^{2}-\frac{E_{n, 1} q_{0}^{2}}{D_{e}}-2 q_{0}\left(e^{\alpha r_{e}}+q_{1}\right)\left(1+e^{\alpha r_{e}}+q_{1}\right)\right)+1(1+1) C_{0} q_{0}^{2}, \\
& Q=\frac{4 \mu D_{e}}{\alpha^{2} \mathrm{~h}^{2}}\left(\frac{E_{n, 1} q_{0}}{D_{e}}-q_{0}+e^{\alpha r_{e}}+q_{1}\right)-1(1+1)\left(1+2 q_{0} C_{0}\right), \\
& R=\frac{2 \mu\left(D_{e}-E_{n, 1}\right)}{\alpha^{2} \mathrm{~h}^{2}}+1(1+1) C_{0} .
\end{aligned}
$$

Comparing Eq. (10) with Eq. (3), the parametric constants in Eq. (6) are obtain as follows

$$
\left.\begin{array}{l}
\alpha_{1}=1, \alpha_{2}=\alpha_{3}=-q_{0}, \alpha_{4}=0, \alpha_{5}=\frac{q_{0}}{2}, \\
\alpha_{6}=q_{0}^{2}\left(\frac{1}{4}+1(1+1) C_{0}\right)+\frac{2 \mu D_{e}}{\alpha^{2} \mathrm{~h}^{2}}\left(q_{0}^{2}-2 q_{0}\left(e^{\alpha r_{e}}+q_{1}\right)-\frac{E_{n, 1} q_{0}^{2}}{D_{e}}+e^{\alpha r_{e}}+q_{1}\right), \\
\alpha_{7}=\frac{4 \mu D_{e}}{\alpha^{2} \mathrm{~h}^{2}}\left(q_{0}-\frac{E_{n, 1} q_{0}}{D_{e}}-e^{\alpha r_{e}}-q_{1}\right)+1(1+1)\left(1+2 q_{0} C_{0}\right), \\
\alpha_{8}=\frac{2 \mu\left(D_{e}-E_{n, 1}\right)}{\alpha^{2} \mathrm{~h}^{2}}+1(1+1) C_{0}, \alpha_{9}=\frac{1}{4}\left(q_{0}^{2}+\frac{8 \mu D_{e}\left(e^{\alpha r_{e}}+q_{1}\right)^{2}}{\alpha^{2} \mathrm{~h}^{2}}\right)-41(1+1) q_{0}, \\
\alpha_{10}=1+2 T_{V_{1}}, \alpha_{11}=-2 q_{0}\left[1+T_{V_{1}}\right]+T_{V_{2}}, \alpha_{12}=T_{V_{1}}, \alpha_{13}=\frac{q_{0}}{2}-\frac{1}{2} T_{V_{2}}+q_{0} T_{V_{1}}, \\
T_{V_{1}}=\sqrt{\frac{2 \mu\left(D_{e}-E_{n, 1}\right)}{\alpha^{2} \mathrm{~h}^{2}}+1(1+1) C_{0}, T_{V_{2}}}=\sqrt{q_{0}^{2}-41(1+1) q_{0}+\frac{8 \mu D_{e}\left(e^{\alpha r_{e}}+q_{1}\right)^{2}}{\alpha^{2} \mathrm{~h}^{2}}}
\end{array}\right\} .
$$

Substituting the parameters in Eq. (14) into Eq. (4), we have the energy equation for the system as

$$
E_{n, 1}=D_{e}+\frac{1(1+1) C_{0} \alpha^{2} h^{2}}{2 \mu}-\frac{\alpha^{2} h^{2}}{2 \mu}\left[\frac{\frac{4 \mu D_{e}\left(e^{\alpha r_{e}}+q_{1}\right)}{\alpha^{2} h^{2}}-1(1+1)-\frac{n(n+1)}{q_{0}^{-1}}+\frac{q_{0}}{2}-\left(n+\frac{1}{2}\right) \lambda_{T}}{\lambda_{T}-q_{0}(2 n+1)}\right]^{2},
$$




$$
\lambda_{T}=\sqrt{q_{0}^{2}-41(1+1) q_{0}+\frac{8 \mu D_{e}\left(e^{\alpha r_{e}}+q_{1}\right)^{2}}{\alpha^{2} \mathrm{~h}^{2}}}
$$

and the corresponding wave function is obtain when the values of $\alpha_{10}$ to $\alpha_{13}$ in Eq. (6) are substituted into Eq. (5),

$$
U_{n, 1}(y)=N_{n, 1} y^{T_{V_{1}}}\left(1+q_{0} y\right)^{T_{V_{1}}\left(q_{0}-1\right)+\frac{1}{2}\left(T_{V_{2}}-q_{0}\right)}\left[P_{n}^{\left(2 T_{V_{1}},-\frac{T_{V_{2}}}{q_{0}}\right)}\left(1+2 q_{0} y\right)\right]
$$

\section{Expectation Values}

In this section, we calculated some expectation values using Hellmann-Faynman Theorey (HFT) [53-57]. When a Hamiltonian $H$ for a given quantum system is a function of some parameter $v$, the energy-eigenvalue $E_{n, 1}$ and the eigenfunction $U_{n, 1}(v)$ of $H$ are given by

$$
\frac{\partial E_{n, 1}(v)}{\partial v}=\left\langle U_{n, 1}(v)\left|\frac{\partial H(v)}{\partial v}\right| U_{n, 1}(v)\right\rangle
$$

with the effective Hamiltonian as

$$
H=-\frac{\mathrm{h}^{2}}{2 \mu} \frac{d^{2} U_{n 1}(r)}{d r^{2}}+\frac{\mathrm{h}^{2}}{2 \mu} \frac{1(1+1)}{r^{2}}+D_{e}-\frac{D_{e}}{e^{-\alpha r}+q_{0}}\left(e^{\alpha r_{e}}+q_{1}-\frac{\left(e^{\alpha r_{e}}+q_{1}\right)^{2}}{e^{-\alpha r}+q_{0}}\right) .
$$

Setting $v=D_{e}, v=1$ and $v=\mu$, we have the expectation values of $p^{2}, r^{-2}$ and $V$ respectively are

$$
\begin{gathered}
\left\langle p^{2}\right\rangle_{n, 1}=1-\frac{4 \alpha^{2} h^{2} A_{2}\left(e^{\alpha r_{e}}+q_{1}\right)\left(\frac{\mu \lambda_{T}-(n+1 / 2)\left(e^{\alpha r_{e}}+q_{1}\right) \mu}{\alpha^{2} h^{2} \lambda_{T}}\right)}{\mu A_{1}^{2}}, \\
\left\langle r^{-2}\right\rangle_{n, 1}=\frac{\mu B}{\mathrm{~h}^{2}(21+1)}+\frac{2 \alpha^{2} \lambda_{T}\left(21+1+B_{0}\right)}{(21+1) A_{1}^{2}}-\frac{4 q_{0} \alpha^{2}}{\lambda_{T} A_{1}}, \\
\langle V\rangle_{n, 1}=\frac{\alpha^{2} h^{2} A_{2}^{2}}{2 \mu^{2} A_{1}^{2}}+\frac{4 D_{e} A_{2}^{2}\left(e^{\alpha r_{e}}+q_{1}\right)^{2}}{\mu \lambda_{T} A_{1}^{2}}-\frac{1(1+1) C_{0} \alpha^{2} h^{2}}{2 \mu^{2}}-\frac{4 D_{e} A_{2}\left(e^{\alpha r_{e}}+q_{1}\right)^{2}\left(\frac{1-(n+1 / 2)\left(e^{\alpha r_{e}}+q_{1}\right)}{\lambda_{T}}\right)}{\mu A_{1}^{2}}, \\
A_{0}=\lambda_{T}(n+1 / 2), \\
A_{1}=\lambda_{T}-q_{0}(1+2 n),
\end{gathered}
$$




$$
\begin{gathered}
A_{2}=\frac{4 \mu D_{e}\left(e^{\alpha r_{e}}+q_{1}\right)}{\alpha^{2} \mathrm{~h}^{2}}+\frac{q_{0}}{2}-\frac{n(n+1)}{q_{0}}-(n+1 / 2) \lambda_{T}-1(1+1), \\
B=\frac{\alpha^{2} \mathrm{~h}^{2}(21+1) C_{0}}{2 \mu}, \\
B_{0}=-\frac{q_{0}(2 n+1)(21+1)}{\lambda_{T}} .
\end{gathered}
$$

Table 1: Comparison of theoretical values with experimental values for the vibrational energy levels of the modified deformed exponential-type molecular potential for $3^{3} \sum_{g}^{+}$state of cesium dimer.

\begin{tabular}{|c|cc|cc|}
\hline$n$ & RKR [58] & $q_{1}=1$ & RKR [51] & $q_{1}=-1$ \\
& $\mathrm{~cm}^{-1}$ & $\mathrm{~cm}^{-1}$ & $\mathrm{~cm}^{-1}$ & $\mathrm{~cm}^{-1}$ \\
\hline 0 & 14.4248 & 14.42647874 & $19,477.5507$ & $19,477.55769$ \\
1 & 43.1680 & 43.17554991 & $19,506.2939$ & $19,506.29999$ \\
2 & 71.7657 & 71.77608344 & $19,534.8916$ & $19,534.90041$ \\
3 & 100.2211 & 100.2450879 & $19,563.3470$ & $19,563.35986$ \\
4 & 128.5375 & 128.5580953 & $19,591.6634$ & $19,591.68592$ \\
5 & 156.7182 & 156.7410068 & $19,619.8441$ & $19,620.43756$ \\
6 & 184.7663 & 184.7735524 & $19,647.8922$ & $19,648.55903$ \\
7 & 212.6851 & 212.6619860 & $19,675.8110$ & $19,677.08704$ \\
8 & 240.4778 & 240.4268832 & $19,703.6037$ & $19,704.38389$ \\
9 & 268.1477 & 268.2399412 & $19,731.2736$ & $19,732.74500$ \\
10 & 295.6980 & 296.0578830 & $19,758.8239$ & $19,759.62187$ \\
11 & 323.1320 & 323.3428999 & $19,786.2579$ & $19,786.99999$ \\
12 & 350.4529 & 351.0106935 & $19,813.5788$ & $19,814.29857$ \\
\hline
\end{tabular}

Table 2: Comparison of theoretical values with experimental (in $\mathrm{cm}^{-1}$ ) values for the vibrational energy levels of the modified deformed exponential-type molecular potential for $5^{1} \Delta_{g}$ state sodium dimer and $a^{3} \Sigma_{u}^{+}$state of lithium dimer

\begin{tabular}{|l|lc|cc|}
\hline$n$ & $\begin{array}{c}|c| \\
{[18]}\end{array}$ & $\begin{array}{c}q_{0}=q_{1}=1 \\
\text { present results }\end{array}$ & $\begin{array}{c}L i_{2} \\
{[18]}\end{array}$ & $\begin{array}{c}q_{0}=q_{1}=-1 \\
\text { present results }\end{array}$ \\
\hline 0 & 60.333 & 60.31364676 & 31.8570 & 31.80143202 \\
1 & 180.373 & 180.2577331 & 90.4530 & 90.39261096 \\
2 & 299.555 & 299.3324068 & 142.523 & 142.4220678 \\
3 & 417.871 & 417.3016667 & 188.240 & 188.1901675 \\
4 & 535.313 & 534.5243524 & 227.679 & 227.4621906 \\
5 & 651.872 & 650.4688333 & 260.837 & 260.5360334 \\
6 & 767.539 & 765.3824841 & 287.665 & 287.3995111 \\
7 & 882.305 & 879.7021966 & 308.098 & 307.9601021 \\
\hline
\end{tabular}




\begin{tabular}{|c|ll|ll|}
\hline 8 & 996.162 & 993.6386433 & 322.155 & 322.2963805 \\
9 & 1109.100 & 1104.753548 & 330.170 & 330.7599572 \\
10 & 1221.113 & 1215.707839 & 333.269 & 333.5586499 \\
\hline
\end{tabular}

Table 3: Expectation values at various quantum states with $1=\mathrm{h}=\mu=1, r_{e}=0.4, \alpha=0.25$, $D_{e}=5$ and $q_{0}=q_{1}=-1$.

\begin{tabular}{|c|ccc|}
\hline$n$ & $\left\langle p^{2}\right\rangle_{n, 1}$ & $\left\langle r^{-2}\right\rangle_{n, 1}$ & $\langle V\rangle_{n, 1}$ \\
\hline 0 & 1.069858799 & 0.039999669 & 0.368447529 \\
1 & 1.051727481 & 0.029330271 & 0.260041512 \\
2 & 1.030363793 & 0.023397637 & 0.133941612 \\
3 & 1.013262325 & 0.019620392 & 0.043515090 \\
4 & 1.000377469 & 0.017004496 & -0.004319914 \\
5 & 0.990813897 & 0.015085714 & -0.010405957 \\
6 & 0.983773583 & 0.013618148 & 0.023299645 \\
7 & 0.978655270 & 0.012459383 & 0.095013696 \\
8 & 0.975020088 & 0.011521231 & 0.203320911 \\
9 & 0.972547147 & 0.010746167 & 0.347136241 \\
10 & 0.970998984 & 0.010095063 & 0.525631819 \\
\hline
\end{tabular}

Table 4: Expectation values at various quantum states with $1=\mathrm{h}=\mu=1, r_{e}=0.4, \alpha=0.25$, $D_{e}=5$ and $q_{0}=q_{1}=1$.

\begin{tabular}{|c|ccc|}
\hline$n$ & $\left\langle p^{2}\right\rangle_{n, 1}$ & $\left\langle r^{-2}\right\rangle_{n, 1}$ & $\langle V\rangle_{n, 1}$ \\
\hline 0 & 12.54126260 & 0.004909147 & 261.9950729 \\
1 & 14.07512966 & 0.005000995 & 285.8523651 \\
2 & 15.80688031 & 0.005100467 & 312.2648238 \\
3 & 17.77133204 & 0.005208552 & 341.6413784 \\
4 & 20.01151893 & 0.005326420 & 374.4812757 \\
5 & 22.58116336 & 0.005455464 & 411.3997764 \\
6 & 25.54806604 & 0.005597350 & 453.1629487 \\
7 & 28.99882749 & 0.005754097 & 500.7354772 \\
8 & 33.04553492 & 0.005928168 & 555.3474242 \\
9 & 37.83541012 & 0.006122603 & 618.5891346 \\
10 & 43.56501898 & 0.006341198 & 692.5488475 \\
\hline
\end{tabular}

The average deviation of the calculated results from the experimental results is obtained using the formula 


$$
\sigma_{a v}=\frac{100}{N} \sum_{n}\left|\frac{E_{E R}-E_{C R}}{E_{E R}}\right|,
$$

where $E_{E R}$ is the sum of the experimental data, $E_{C R}$ is the sum of the calculated values and $N$ is the total number of the experimental data.

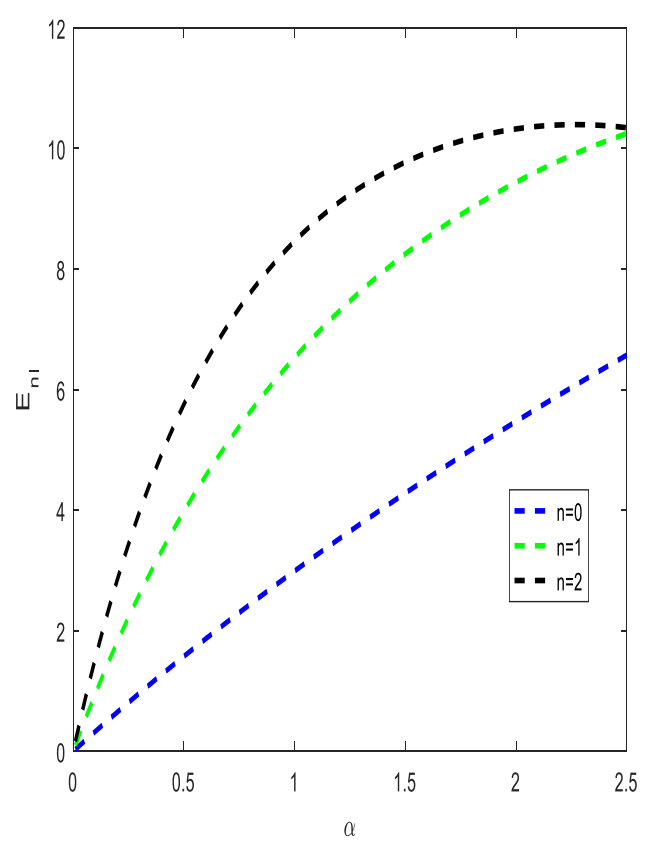

(a) $q_{0}=q_{1}=1$

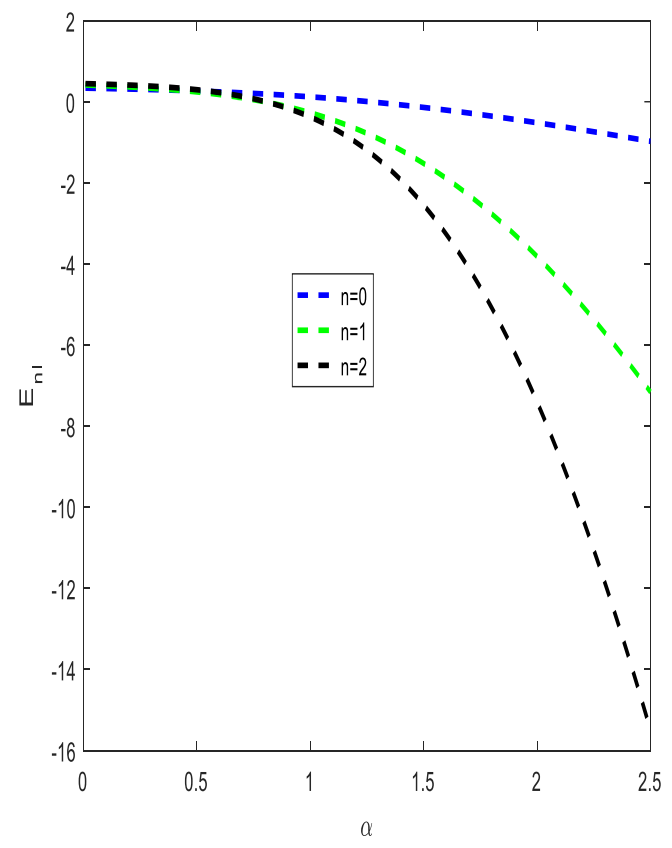

(b) $q_{0}=q_{1}=-1$

Figure 1: Variation of energy $E_{n, 1} \quad$ against the screening parameter $\alpha$, with $C=1=\mathrm{h}=\mu=1, r_{e}=0.4$ and $D_{e}=10$.

\section{Discussion of Result}

The comparison of the observed values of RKR and calculated values for $3^{3} \Sigma_{g}^{+}$state of cesium dimer with $q_{0}=q_{1}=1, q_{0}=q_{1}=-1, D_{e}=2722.28 \mathrm{~cm}^{-1}, r_{e}=5.3474208 \stackrel{0}{A}$, and $\omega_{e}=28.891 \mathrm{~cm}^{-1}$ is reported in Table 1. The results for two values for each of the deformed parameter and adjustable parameter agreed with the observed values of the cesium dimer. In Table 2, the comparison of vibrational energies of sodium dimer and lithium dimer respectively are reported. When the deformed parameter and the adjustable parameter are taken as one with $D_{e}=79885 \mathrm{~cm}^{-1}$, $r_{e}=1.097 \stackrel{0}{A}, \omega_{e}=2358.6 \mathrm{~cm}^{-1}$, the results agreed with the observed values of $5^{1} \Delta_{g}$ state of 
sodium dimer. Taken the deformed parameter and adjustable parameter respectively as minus one, with $D_{e}=2722.28 \mathrm{~cm}^{-1}, r_{e}=4.173 \stackrel{0}{A}$ and $\omega_{e}=65.130 \mathrm{~cm}^{-1}$, the results obtained correspond to the observed values of lithium dimer.

To deduce the effect of the deformed and adjustable parameters on the numerical values and discrepancy of the calculated results from the experimental data, we used the formula given in Eq. (28). For cesium dimer, the average percentage deviation for $q_{0}=q_{1}=1$ is $0.0038 \%$ while the average percentage deviation for $q_{0}=q_{1}=-1$ is $0.0002 \%$. For sodium dimer with $q_{0}=q_{1}=1$, the average percentage deviation is $0.0283 \%$ while the average percentage deviation for lithium dimer with $q_{0}=q_{1}=-1$, is $0.0010 \%$. In Table 3 and Table 4, we presented the numerical results for the three different expectation values calculated in Eq. (20), Eq. (21) and Eq. (22) respectively. The results obtained with $q_{0}=q_{1}=1$ are higher than their counterpart obtained with $q_{0}=q_{1}=-1$.

The effect of the screening parameter on the energy eigenvalues with two values each of the deformed parameter and adjustable parameter is shown in Figure 1. For $q_{0}=q_{1}=1$, the energy of the system rises as the screening parameter increases from zero but the revise is the case for $q_{0}=q_{1}=-1$.

\section{Conclusion}

The solutions of a one-dimensional Schrödinger equation is obtained for a molecular potential model using parametric Nikiforov-Uvarov method. By changing the numerical values of the deformed parameter and adjustable parameter, the results different obtained for different molecules agreed with experimental values. However, the results obtained with $q_{0}=q_{1}=-1$ are closer to the experimental values compared with the results obtained with $q_{0}=q_{1}=1$. In the computation of numerical values for the expectation values, it is noted that the results obtained with $q_{0}=q_{1}=1$ are higher than their counterpart obtained with $q_{0}=q_{1}=-1$.

\section{Declarations}

Funding: N/A

Conflict of interest/competing interest: N/A

Availability of data and material: N/A

Code availability: N/A

Authors' contributions: C.A. Onate; Formulate the work, solved the calculations

I.B. Okon; Wrote the introduction

M.C. Onyeaju; Makes all the plots and discussed the results

O. Ebomwonyi; Solved the calculations and typeset the paper

\section{Reference}


[1] Hu, X. T., Liu, J. Y. \& Jia, C. S. The $3^{3} \Sigma_{g}^{+}$state of $C s_{2}$ molecule. Comput. Theor, Chem. 1019, 137 (2013).

[2] Lino da Silva, M., Guerra, V., Loureiro, J. \& Sá, P. A. Vibrational distributions in N2 with an improved calculation of energy levels using the RKR method. Chem. Phys. 348, 187 (2008).

[3] Eg rifes, H., Demirhan, D. \& Buyukk1lıc, F. Exact solutions of the Schrödinger equation for the deformed hyperbolic potential well and the deformed four-parameter exponential type potential. Phys. Lett. A 275, 229 (2000).

[4] Horchani, R., Al-Kindi, N. \& Jelassi, H. Ro-vibrational energies of caesium molecules with the Tietz-Hua oscillator. Molecular Physics (2020), DOI: 10.1080/00268976.2020.1812746

[5] Whang, T. \& Cheng, C. -P. Observation of $L$ uncoupling in the $5^{1} \Delta_{g}$ Rydberg state of $N a_{2}$

J. Chem. Phys, 123, 224303 (2005).

[6] Farout, M., Bassalat, A. \& Ikhdair, S. M. Exact quantized momentum eigenvalues and eigenstates of a general potential model. J. Appl. Math. Phys. 8, 1434 (2020).

[7] Farout, M., Bassalat, A. \& Ikhdair, S. M. Feinberg-Horodecki exact momentum states of improved deformed exponential-type potential. J. Appl. Math. Phys. 8, 1496 (2020).

[8] Jia, C. -S., Diao, Y. -F., Liu, X. -J., Wang, P. -Q., Liu, J. -Y. \& Zhang, G. -D. Equivalence of the Wei potential model and Tietz potential model for diatomic molecules. J. Chem. Phys. 137, 014101 (2012).

[9] Liu, J. -Y., Hu, X. -T. \& Jia, C. -S. Molecular energies of the improved Rosen- Morse potential energy model. Can. J. Chem. 92, 40 (2014).

[10] Song, X. -Q., Wang, C.-W. \& Jia, C. -S. Thermodynamics properties of sodium dimer. Chem. Phys. Lett. 673, 50 (2017).

[11] Hamzavi, M., Rajabi, A. A. \& Thylwe, K. -E. The rotation-vibration spectrum of diatomic molecules with the tietz-hua rotating oscillator. Int. J. Quant. Chem. 112, 2701 (2012)

[12] Falaye, B. J., Oyewumi, K. J., Ikhdair, S. M. \& Hamzavi, M. Eigensolution techniques, their applications and Fisher's information entropy of the Tietz-Wei diatomic molecular model. Phys. Scr. 89, 115204 (2014).

[13] Onyeaju, M. C. \& Onate, C. A. Vibrational Entropy and Complexity Measures in Modified Pöschl-Teller plus Woods-Saxon potential. Few-Body Syst. 61, 21 (2020).

[14] Okorie, U. S., \& Ibekwe, E. E., Onyeaju, M. C. \& Ikot, A. N. Solutions of the Dirac and Schrödinger equations with shifted Tietz-Wei potential. Eur. Phys. J. Plus 133, 433 (2018).

[15] Onate, C. A., Adebiyi, L. S. \& Bankole, D. T. Eigensolutions and theoretic quantities under the nonrelativistic wave equation. J. Theor. Comput. Chem.19, 2050007 (2020).

[16] Nikiforov, A. F. \& Uvarov, V. B. Special Functions of Mathematical Physics. Birkhäuser, Basel (1988)

[17] Tezcan, C. \& Sever, R. A general approach for the exact solution of the Schrödinger equation.

Int. J. Theor. Phys. 48, 337 (2009).

[18] Bayrak, O. \& Boztosun, I. Bound state solutions of the Hulthén potential by using the asymptotic iteration method. Phys. Scr. 76, 92 (2007).

[19] Witten, E. Dynamical breaking of supersymmetry. Nucl. Phys. B 188, 513 (1981).

[20] Cooper, F., Khare, A. \& Sukhatme, U. Supersymmetry and quantum mechanics. Phys. Rep. 251, 267 (1995). 
[21] Dong, S. -H. Factorization Method in Quantum Mechanics, Springer Kluwer Academic (2007).

[22] Ma, Z. -Q. \& Xu, B. -W. Quantum correction in exact quantization rules. Europhys. Lett. 69, 685 (2005)

[23] W.-C, S.-H. Dong, S. -H. Proper quantization rule. EPL 89, 10003 (2010).

[24] Ikot, A. N., Okorie, U. S., Rampho, G. J. \& Amadi, P. O. Approximate Analytical Solutions of the Klein-Gordon Equation with Generalized Morse Potential. Int. J. Thermophys. 42, 10 (2021).

[25] Ikot, A. N., Okorie, U. S., Amadi, P. O., Edet, C. O., Rampho, G. J. \& Sever, R. The Nikiforov-Uvarov-Functional Analysis (NUFA) Method: A New Approach for Solving Exponential-Type Potential. Few Body Syst. 62, 1 (2021).

[26] Dong, S. -H. \& Gu, X. Y. Arbitrary 1 state solutions of the Schrödinger equation with the Deng-Fan molecular potential. J. Phys. Conference Series 96, 012109 (2008).

[27] Zhang, L. H., Li, X. P. \& Jia, C. -S. Approximate solutions of the Schrödinger equation with the generalized Morse potential model including the centrifugal term. Int. J. Quant. Chem. 111, 1870 (2011).

[28] Onate, C. A., Ikot, A. N., Onyeaju, M. C., Ebomwonyi, O. \& Idiodi, J. O. A.. Effect of dissociation energy on Shannon and Rẻnyi entropies. Karbala Int. J. Mod. Scien. 4 (2018) 134142

[29] Najafizade, S. A., Hassanabadi, H. \& Zarrinkamar, S. Nonrelativistic Shannon information entropy for Kratzer potential. Chin. Phys. B 25, 040301 (2016).

[30] Ghafourian, M. \& Hassanabadi, H. Shannon information entropies for the three-dimensional Klein-Gordon problem with the Poschl-Teller potential. J. Korean Phys. Soc. 68, 1267 (2016).

[31] Boumali, A. \& Labidi, M. Shannon entropy and Fisher information of the one-dimensional Klein-Gordon oscillator with energy-dependent potential. Mod. Phys. Lett. A 33, 1850033 (2018).

[32] Idiodi, J. O. A. \& Onate, C. A. Entropy, Fisher Information and Variance with FrostMusulin Potential. Commum. Theor. Phys. 66, 269 (2016).

[33] Okorie, U. S., Ikot, A. N., Chukwuocha, E. O. \& Rampho, G. J. Thermodynamic properties of improved deformed exponential-type potential (IDEP) for some diatomic molecules. Results in Phys. 17, 103078 (2020).

[34] Khordad, R. \& Ghanbari, A. Theoretical Prediction of Thermodynamic Functions of TiC: Morse Ring-Shaped Potential. Low Temp. Phys. 199, 1198 (2020).

[35] Wang, J., Jia, C.S., Li, C. J., Peng, X. L., Zhang, L. H. \& Liu, J. Y. Thermodynamic Properties for Carbon Dioxide. ACS Omega 4, 19193 (2019).

[36] Onate, C. A. \& Ojonubah, J. O. Relativistic and nonrelativistic solutions of the generalized Pöschl-Teller and hyperbolical potentials with some thermodynamic properties. Int. J. Mod. Phys. E 24, 1550020 (2015).

[37] Yahya, W. A. \& Oyewumi, K. J. Thermodynamic properties and approximate solutions of the $\ell$-state Pöschl-Teller-type potential. J. Ass. Arab. Univ. Basic Appl. Scien. 21, 53 (2016).

[38] K.J. Oyewumi, B.J. Falaye, C.A. Onate, O.J. Oluwadare, W.A. Yahya. Thermodynamic properties and the approximate solutions of the Schrödinger equation with the shifted Deng-Fan potential model. Mol. Phys. 112, 127 (2014)

[39] Mesa, A. D. S., Quesne, C. \& Smirnov, Y. F. Generalized Morse potential: Symmetry and satellite potentials. J. Phys. A: Math. Theor. 31, 321 (1998). 
[40] Rong, Z., Kjaergaard, H. G. \& Sage, M. L. Comparison of the Morse and Deng-Fan potentials for X-H bonds in small molecules. Mol. Phys. 101, 2285 (2003)

[41] Gordillo-Vazquez, F. J. \& Kunc J. A. Comparison of fluorescence-based temperature sensor schemes: Theoretical analysis and experimental validation. J. Appl. Phys. 84, 4649 (1998).

[42] Chackerian, C. \& Tipping, R. H. Vibration-rotational and rotational intensities for CO isotopes J. Mol. Spectrosc. 99, 431 (1983)

[43] Manai, I., Horchani, R., Lignier, H., Pillet, P. \& Comparat, D. Rovibrational cooling of molecules by optical pumping. Phys. Rev. Lett. 109, 183001 (2012)

[44] Viteau, M., Chotia, A., Allegrini, M., Bouloufa, N, Dulieu, O., D. Comparat, D. \& Pillet, P. Optical pumping and vibrational cooling of molecules. Science 321, 232 (2008).

[45] Vala, J., Dulieu, O., Masnou-Seeuws, F., Pillet, P. \& Kosloff, R. Coherent control of coldmolecule formation through photoassociation using a chirped-pulsed-laser field. Phys. Rev. A 63, 013412 (2000).

[46] Fioretti, A., Sofikitis, D., Horchani, R., Li, X., Pichler, M., Weber, S., Allegrini, M., Chatel, B., Comparat, D. \& Pillet, P. Cold cesium molecules: from formation to cooling. J. Mod. Opt. 56, 2089 (2009)

[47] Vatasescu. M. Preparing isolated vibrational wave packets with light-induced molecular potentials by chirped laser pulses. Nucl. Instrum. Methods B 279, 8 (2012).

[48] Beloy, K., Borschevsky, A., Flambaum, V. V. \& Schwerdtfeger, P. Effect of $\alpha$ variation on a prospective experiment to detect variation of $\mathrm{me} / \mathrm{mp}$ in diatomic molecules. Phys. Rev. A 84, 042117 (2011).

[49] Li, D., Xie, F. \& Li, L. Observation of the $C s_{2}\left(3^{3} \Sigma_{g}^{+}\right)$state by infrared-infrared double resonance. Chem. Phys. Lett. 458, 267 (2008).

[50] Li, D., Xie, F., Li, L., Magnier, S., Sovkov, V. B. \& Ivanov, V. S. The $3^{3} \Sigma_{g}^{+}$and a $3 \Sigma_{u}^{+}$ states of $\mathrm{Cs}_{2}$ : Observation and calculation. Chem. Phys. Lett. 441, 39 (2007)

[51] Li, D., Xie, F. \& Li, L. Observation of the $\mathrm{Cs}_{2}\left(3^{3} \Sigma_{g}^{+}\right)$state by infrared-infrared double resonance. Chem. Phys. Lett. 458, 267 (2008).

[52] Onate, C. A. \& Idiodi, J. O. A. Fisher Information and Complexity Measure of Generalized Morse Potential Model. Commun. Theor. Phys. 66, 275 (2016)

[53] Jia, C. -S., Liu, J. -Y., Wang, P. -Q. \& Lin, X. Approximate analytical solutions of the Dirac equation with the hyperbolic potential in the presence of the spin symmetry and pseudospin symmetry. Int. J. Theor. Phys. 48, 2633 (2009).

[54] Ikot, A. N., Okorie, U. S., Rampho, G. J., Amadi, P. O., Edet, C. O., Akpan, I. O., Abdullah, H. Y. \& Horchani, R. Klein-Gordon Equation and Nonrelativistic Thermodynamic Properties with Improved Screened Kratzer Potential. J. Low Temp. Phys. Doi: 10.1007/S10909-02002544-w

[55] Dong, S. -H., Lozada-Cassou. M., Yu, J., Jiménez-Ấngeles, F. \& Rivera, A. L. Hidden symmetries and thermodynamic properties for a harmonic oscillator plus an inverse square potential. Int. J. Quant. Chem. 107, 366 (2007).

[56] Eshghi, M., Mehraban, H. \& Ikhdair, S. M. Approximate energies and thermal properties of a position-dependent mass charged particle under external magnetic fields. Chin. Phys. B 26, 060302 (2017).

[57] Okorie, U. S., Edet, C. O., Ikot, A. N., Rampho, G. J. \& Sever, R. Thermodynamic functions for diatomic molecules with modified Kratzer plus screened Coulomb potential. Indian J. Phys. 95, 411 (2021) 
[58] Hu, X. -T., Liu, J. - Y. \& Jia, C. -S. The $3^{3} \Sigma_{g}^{+}$state of $C s_{2}$ molecule. Comput. Theor. Chem. 1019, 137 (2013). 